\title{
JUURNAL.RU
}

COMPANY GROUP "INTELLEKT"

Безрукова А.А. ФГБОУ ВО НГТУ Новосибирск, Россия

doi: 10.18411/lj2016-5-4-01

\section{Предельные законы управления: сравнительный анализ при действии помехи измерения}

\begin{abstract}
Аннотация:
В статье представлен сравнительный анализ систем, в которых использовались регуляторы со старшей производной в управлении, с ограниченным коэффициентом и со скользящим режимом. Проведено исследование процессов в системах с различными регуляторами при изменении различных параметров системы и наличии аддитивной помехи измерения на выходной величине. Моделирование проводилось в программной среде Matlab Simulink.

Ключевые слова: старшая производная в управлении, релейный закон управления, скользящий режим, большой коэффициент усиления, дифференцирующий фильтр, помехи измерения.
\end{abstract}

\section{Введение}

Под предельными законами управления понимают такие законы, которые позволяют полностью подавить возмущение и обеспечить эффективную отработку помехи измерения с заданной точностью.

Идея таких законов управления основана на использовании достаточно большого значения коэффициента усиления, которое в пределе обеспечивает выполнение необходимых требований к системе. 
Цель данной работы - сформулировать рекомендации по выбору регулятора на основании выполненных исследований.

\section{1. Постановка задачи}

Рассматривается динамический объект, модель которого имеет вид:

$$
\ddot{y}+a_{1}(t) \dot{y}+a_{2}(t) y=b(t) u,
$$

где коэффициенты $a_{i}$ изменяются в ограниченном диапазоне:

$$
a_{i \min } \leq a_{i}(t) \leq a_{i \max }, i=1,2 .
$$

При номинальных значениях параметров модель (1) может быть описана передаточной функцией:

$$
W(p)=\frac{k_{o}}{T^{2} p^{2}+2 d T p+1},
$$

где $k_{0}$ - коэффициент усиления, Т - постоянная времени, $\mathrm{d}$ - коэффициент демпфирования.

Исследуемая система состоит из объекта управления (2) и регулятора. Необходимо обеспечить выполнение требований к качеству переходного процесса, которые задаются в виде оценок:

$$
t_{\Pi} \leq t_{\Pi}^{*} ; \sigma \leq \sigma^{*} ; \Delta^{0} \leq \Delta_{*}^{0} .
$$

\section{2. Исследуемые регуляторы}

Регулятор со старшей производной в управлении [1] предусматривает использование старшей производной выходного переменной объекта (1). Такой подход позволяет эффективно отрабатывать возмущения и подавлять аддитивные помехи измерения сразу в контуре быстрых движений.

Закон управления для объекта (1) имеет вид:

$$
u(t)=K[F(y, \dot{y})-\ddot{y}]
$$

где $\mathrm{K}-$ коэффициент усиления, $F(y, \dot{y})$ - функция, отражающая требования к динамике системы. Коэффициент регулятора выбирается из условия

$$
\left|b_{\min }\right| K \approx(20 \ldots 100)
$$

в зависимости от значения желаемой статической ошибки $\Delta_{*}^{0}$. 
Замкнутая система с регулятором в таком случае принимает вид:

$$
y=\frac{f(\cdot)}{1+b(\cdot) K}+\frac{b(\cdot) K}{1+b(\cdot) K} F(\cdot) .
$$

Тогда при увеличении коэффициента $\mathrm{K}$ процессы в системе будут стремиться к желаемым.

Идея применения регуляторов с ограниченным коэффициентом и релейным управлением состоит в использовании бесконечно большого коэффициента в малой окрестности [2].

В обеих системах на основе желаемого уравнения формируется поверхность скольжения $S(x)$, от знака которой будет зависеть управляющее воздействие . Поверхность скольжения для объекта (1)определяется как

$$
S(x)=F(y, v)-\dot{y},
$$

где $F(y, v)$ - желаемое уравнение.

Управление для системы с ограниченным коэффициентом имеет вид:

$$
u=\left\{\begin{array}{c}
u_{m}, S \geq s_{0} \\
K S,|S|<s_{0} . \\
-u_{m}, S \leq-s_{0}
\end{array} .\right.
$$

Релейный закон управления описывается как

$$
u=u_{m} \operatorname{sign} S(x)=\left\{\begin{array}{c}
u_{m}, S>0 \\
-u_{m}, S<0
\end{array} .\right.
$$

Реализация всех типов регулятора, рассмотренных в этой работе, предполагает использование оценок производных выходного сигнала. Для обеспечения этого условия в системы в обратную связь включается дифференцирующий фильтр. Для подавления помехи измерения необходимо, чтобы степень фильтрующего полинома была хотя бы на 1 порядок больше степени форсирующего [2].

Для систем с регулятором с ограниченным коэффициентом и релейным законом управления независимо от наличия помех измерения, а также для систем со старшей производной в управлении в отсутствии помехи используется ДФ 2-го порядка вида 


$$
W_{f_{2}}(p)=\frac{1}{\mu^{2} p^{2}+2 d_{f} \mu p+1} .
$$

Для систем со старшей производной в управлении при наличии помехи на выходе применяется ДФ 3-го порядка

$$
W_{f_{3}}(p)=\frac{1}{\mu^{3} p^{3}+d_{2} \mu^{2} p^{2}+d_{1} \mu p+1} .
$$

Численные значения параметров фильтрующего полинома 2-го порядка на практике выбираются по соотношениям:

$$
\left\{\begin{array}{c}
\mu \approx 0.1 T^{*} \\
d_{f}=(0.5 \ldots 0.7)
\end{array}\right.
$$

где $\mathrm{T}^{*}$ - желаемая постоянная времени.

Для нахождения коэффициентов ДФ 3-го порядка необходимо воспользоваться диаграммой Вышнеградского, чтобы обеспечить устойчивость процессов в контуре быстрых движений.

\section{3. Исследование свойств систем}

Свойства систем рассматриваются на примере объекта (2), параметры которого будем изменять в диапазонах: $1,4 \leq k_{o} \leq 2,6 ; 1,05 \leq T \leq$ 1,$95 ; 0,14 \leq d \leq 0,26$. Номинальные значения параметров: $k_{o}=2 ; T=$ 1,$5 ; d=0,2$. Требования к качеству переходных процессов заданы в виде оценок: $t_{\text {п }} \leq 3 c ; \sigma=0 \% ; \Delta^{0} \leq 5 \%$.

Желаемое уравнение 2-го порядка, рассчитанное с использованием модального подхода, для системы со старшей производной в управлении имеет вид:

$$
\ddot{y}=-4 \dot{y}-4 y+4 .
$$

Выберем коэффициент усиления такого регулятора $K_{1}=10$.

Желаемое уравнение 1-го порядка для систем с ограниченным коэффициентом и релейным управлением имеет вид:

$$
\dot{y}=-2 y+2 .
$$

Ограниченный коэффициент усиления выбирается как $K_{2}=10$. Уровни 
ограничения для регуляторов с ограниченным коэффициентом и со скользящим режимом задаются одинаковыми: $u_{m}=1$. Для реле с гистерезисом устанавливаем значения Switch on point: 0,01; Switch off point: 0 .

Параметры дифференцирующего фильтра 2-го порядка в отсутствии помех измерения для всех исследуемых систем: $\mu=0,05 ; d_{f}=0,7$.

Параметры дифференцирующего фильтра 3-го порядка в присутствии помех измерения для системы со старшей производной в управлении: $\mu=$ 0,$05 ; d_{f 1}=d_{f 2}=5 . \quad$ Коэффициенты $\quad d_{f 1}$ и $d_{f 2} \quad$ найдены $\quad$ по критерию устойчивости Гурвица и диаграмме Вышнеградского.

Помеха измерения будет синусоидой с малой амплитудой (1-2\% от уровня основных процессов в системе) и высокой частотой (на порядок больше рабочих частот системы). Параметры помехи измерения: амплитуда $A=0.02$; частота изменяется $\omega_{h}=100$ рад/с.

На Рис. 1 представлены графики переходных процессов в замкнутых системах с регуляторами со старшей производной в управление, с ограниченным коэффициентом и со скользящим режимом.

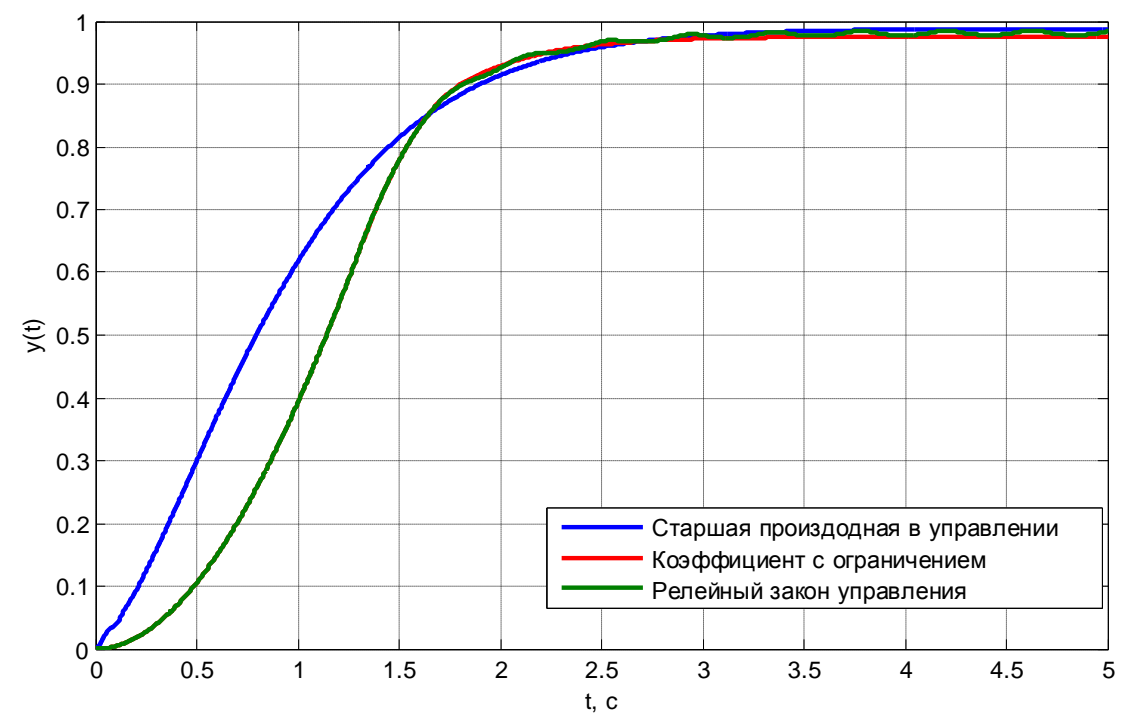

Рис. 1 Переходные процессы в исследуемых системах

Из Рис. 1 видно, что переходные процессы в системах с ограниченным коэффициентом и со скользящим режимом отличаются незначительно, а именно полностью совпадают до момента времени $t=1,26 c$. Это связано с действием 
ограничения на управляющее воздействие, которое фактически размыкает обратную связь в обеих системах. Также можно наблюдать наличие инерционности в регуляторах с коэффициентом ограничения и с релейным управление по сравнению с регулятором со старшей производной в управлении. Bсе переходные процессы соответствуют заданным требованиям к качеству. Следует отметить, что регулятор со старшей производной в управлении немного уступил в быстродействии, однако при его использовании система имела наименьшую статическую ошибку.

Исследуем свойства систем с ограниченным управлением при изменении параметров объекта управления. Для этого увеличим и уменьшим постоянную времени объекта от номинального значения. Изменение этого параметра для системы со старшей производной в управлении практически не влияет на качество переходных процессов. Отработка нестационарных параметров происходит в начальный момент времени. Управляющее воздействие качественно не меняет свой вид. Ресурс управления остается постоянным [3].

Рис. 2 и 3 иллюстрирует переходные процессы в системе с регулятором с ограниченным коэффициентом и графики управляющего воздействия соответственно.

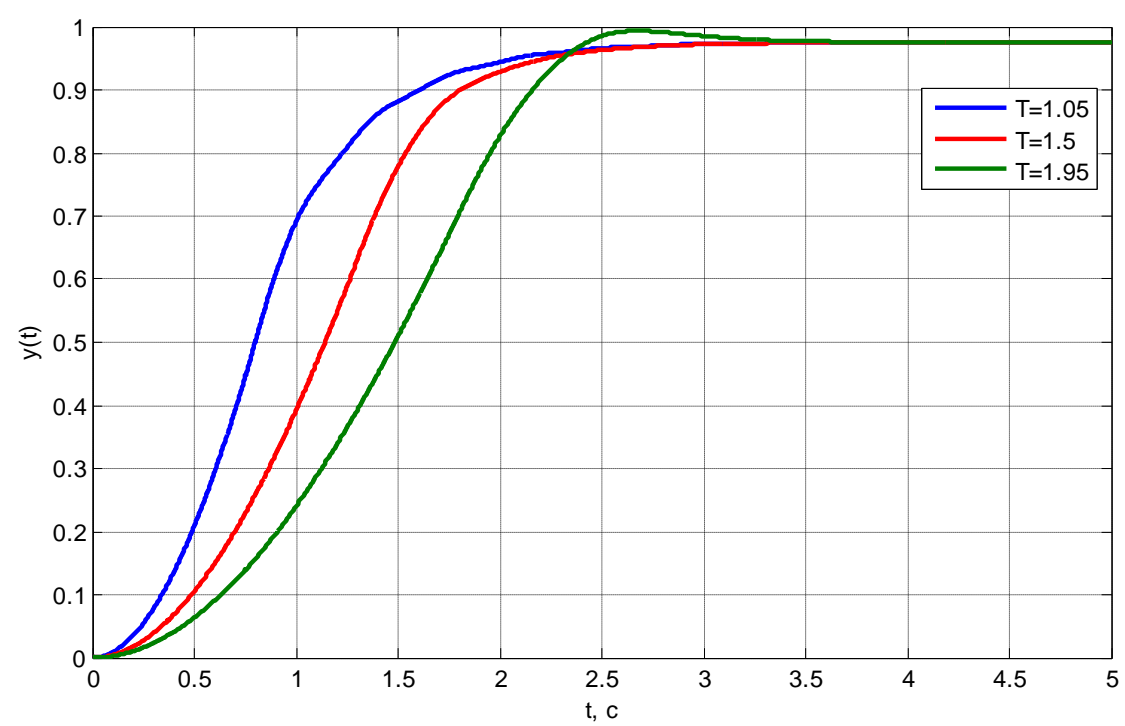

Рис. 2 Влияние постоянной времени объекта Т на переходные процессы в системе с ограниченным коэффициентом 


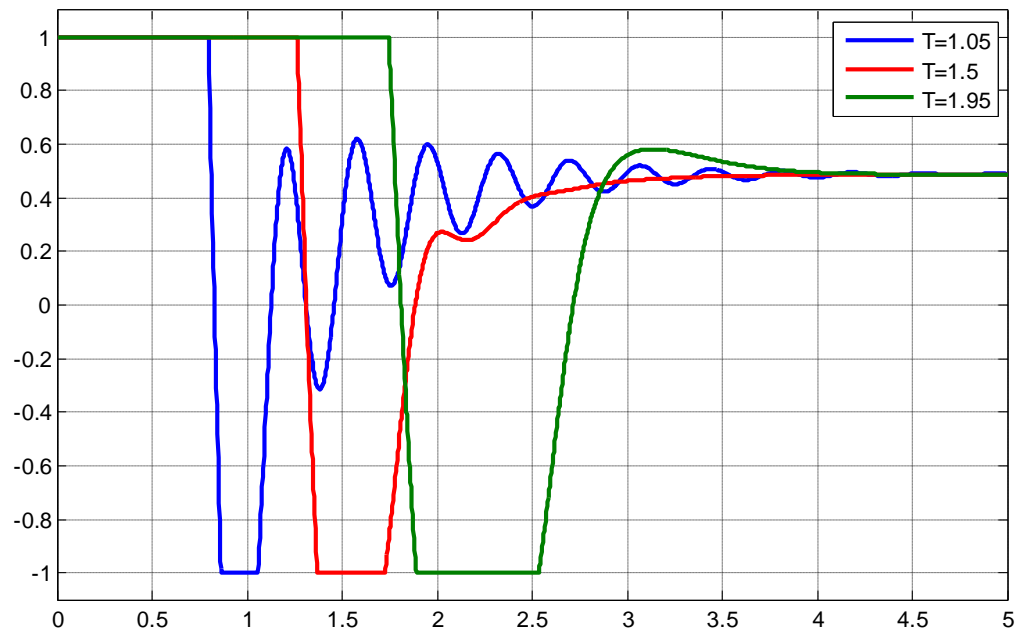

Рис. 3 Влияние постоянной времени объекта Т на управляющее воздействие в системе с ограниченным коэффициентом

Из Рис. 2 видно, что при увеличении постоянной времени объекта Т увеличивается как время переходного процесса, так и перерегулирование в системе. По Рис. 3 можно сделать вывод, что при уменьшении Т возрастает колебательность. Если теперь снять ограничение коэффициента, то можно заметить, что ресурс управления не изменяется и процессы в системе будут не так сильно зависеть от постоянной времени объекта.

На Рис. 4 и 5 представлены аналогичные графики для процессов в системе с релейным управлением.

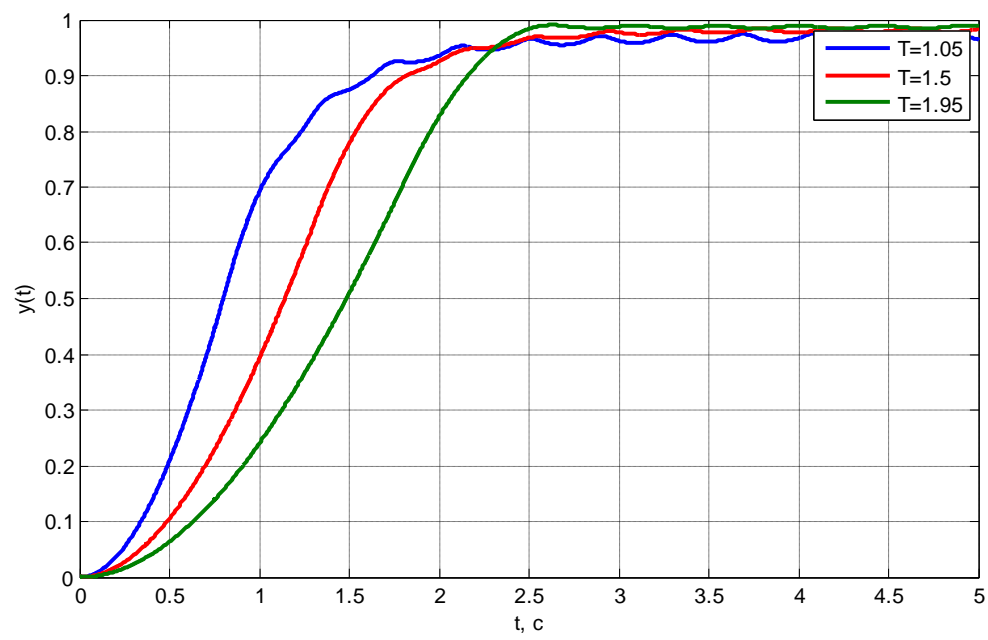

Рис. 4 Влияние постоянной времени объекта Т на переходные процессы в системе со скользящим режимом 

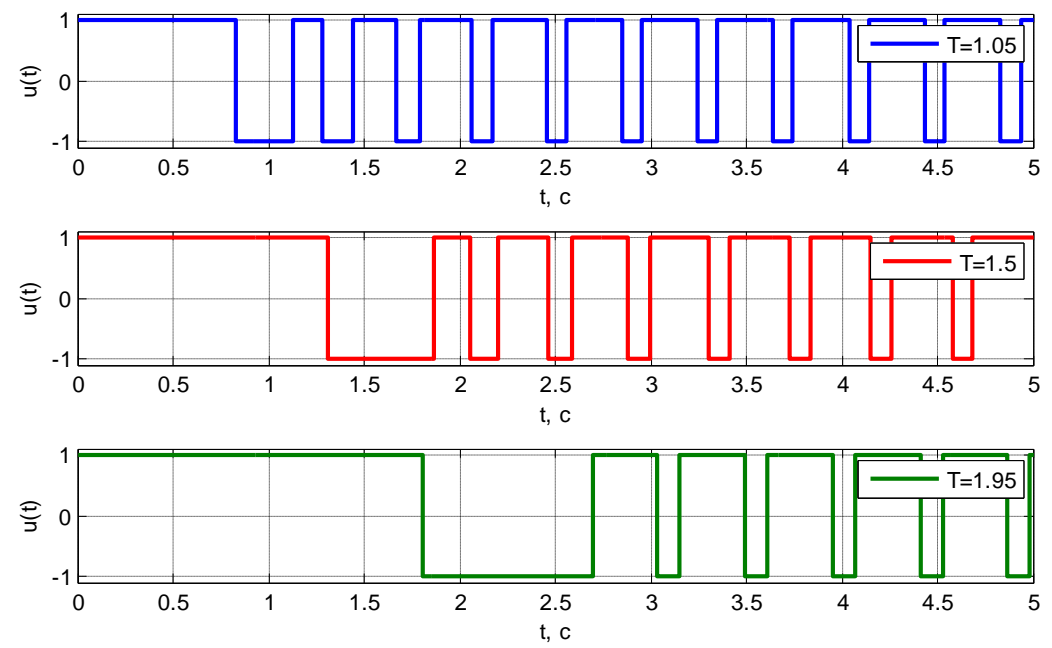

Рис. 5 Влияние постоянной времени объекта Т на управляющее воздействие в системе со скользящим режимом

Результаты моделирования на Рис. 4 и 5 совпадают с результатами для системы с ограниченным коэффициентом - присутствуют те же зависимости выхода системы и управления от постоянной времени объекта. Такая реакция этих двух систем обусловлена наличием одинакового уровня ограничения. То есть время действия разрыва обратной связи для обеих системы одинаковое.

Далее рассматривается работа регуляторов при наличии помехи измерения. Для эффективного подавления помехи в исследуемых системах использовалось правило: $\operatorname{deg} D(\mu p)>\operatorname{deg} C(p)$. Известно, что чем больше частота помехи измерения, тем меньше эта помеха проходит на выходной сигнал, то есть амплитуда помехи уменьшается. Объяснить такую зависимость $y(h)$ можно по амплитудно-частотной характеристике контура быстрых движений, в состав которого и входит фильтр.

Сравним подавление помехи с одинаковыми параметрами для разных систем. Графики переходных процессов для исследуемых систем при действии синусоидальной помехи малой амплитуды и высокой частоты представлены на Рис. 6. 

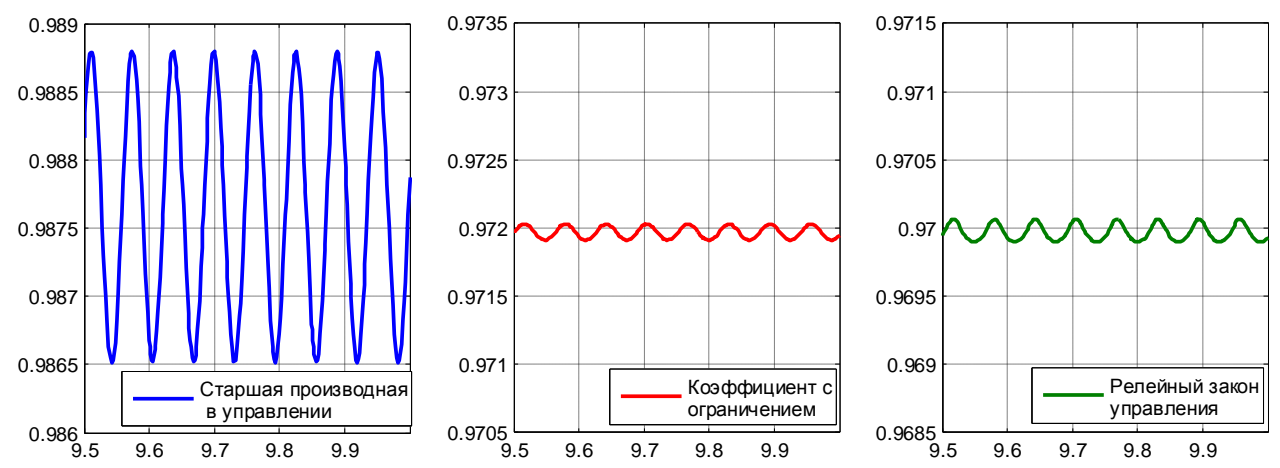

Рис. 6 Графики переходных процессов в системах с различными регуляторами при наличии помехи измерения

Хуже всего с задачей подавления помехи на выходе справляется регулятор со старшей производной в управлении (Рис. 6). Амплитуды помехи для регуляторов с ограниченным коэффициентом и релейным управлением отличается незначительно друг от друга и примерно в 10 раз меньше амплитуды системы со старшей производной в управлении. Стоит отметить, что переходные процессы для всех регуляторов соответствуют ранее заданным требованиям переходного процесса.

\section{Заключение}

По результатам проведенного исследования можно предложить следующие рекомендации по выбору регулятора: если объект содержит нестационарные параметры или его математическая модель не полностью известна, то лучше использовать регулятор со старшей производной в управлении. Регуляторы с ограниченным коэффициентом и релейным управлением в большей степени подвержены изменению параметров объекта в связи с разрывание обратной связи из-за ограничения управления.

Следует отметить, что при известных параметрах объекта переходные процессы во всех исследуемых системах соответствовали требуемому качеству. Однако регулятор со старшей производной в управлении немного уступил в быстродействии, но при его использовании система имела наименьшую статическую ошибку.

По сравнению с регулятором со старшей производной в управлении регуляторы с ограниченным коэффициентом и релейным управление имеет 
недостаток - введение инерционности в систему.

Исследование с введением помехи измерения показало, что рассмотренные регуляторы при правильном выборе дифференцирующего фильтра обеспечивают подавление помехи на выходе при увеличении её частоты. Наиболее успешно с задачей подавления помехи справились регуляторы с ограниченным коэффициентом и релейным законом управления.

Тем не менее, при наличии помехи регулятор со старшей производной в управлении обеспечил статическую ошибку менее 1,5\% в сравнении с 3\% со стороны регуляторов с ограниченным коэффициентом и релейным законом управления, что в некоторых системах гораздо важнее.

Так как исследуемые регуляторы имеют свои преимущества и недостатки, выбор алгоритма управления должен зависит от условий поставленной задачи. 


\section{Литература:}

1. Востриков А.С. Теория автоматического регулирования: Учеб. пособие / Востриков А.С., Французова Г.А. - Новосибирск: Изд-во НГТУ, 2006. 368 с. - («Учебники НГТУ»)

2. Востриков А.С. Задача синтеза в теории регулирования : учеб. пособие / А.С. Востриков. - Новосибирск : Изд-во НГТУ, 2011. - 104 с.

3. Французова Г.А., Толстоконева Е.П, Шилкова Н.Ю. Сравнительный анализ возможностей типовых регуляторов и регуляторов по методу локализации / Автоматика и программная инеженерия. - 2015. - №1(11). - с. 29-34. 